CLINICAL STUDY

\title{
Long-term effects of radiotherapy on cardiovascular risk factors in acromegaly
}

Cristina L Ronchi ${ }^{1,2}$, Elisa Verrua ${ }^{1,3}$, Emanuele Ferrante ${ }^{1,3}$, Gwendolyn Bender ${ }^{2}$. Elisa Sala $^{1,3}$ Andrea G Lania ${ }^{1,3}$ Martin Fassnacht ${ }^{2}$, Paolo Beck-Peccoz ${ }^{1,3}$, Bruno Allolio ${ }^{2}$, Anna Spada ${ }^{1,3}$ and Maura Arosio ${ }^{3,4}$

${ }^{1}$ Unit of Endocrinology and Diabetology, Fondazione IRCCS Ospedale Maggiore Policlinico, 20122 Milan, Italy, ${ }^{2}$ Unit of Endocrinology and Diabetology, Department of Internal Medicine, University Hospital of Wuerzburg, Oberduerrbacher-Strasse 6, 97080 Wuerzburg, Germany, ${ }^{3}$ Department of Medical Sciences, University of Milan, 20122 Milan, Italy and ${ }^{4}$ Unit of Endocrine Diseases and Diabetology, Ospedale San Giuseppe, Multimedica, 20123 Milan, Italy

(Correspondence should be addressed to C L Ronchi at University Hospital of Wuerzburg; Email: cry_ronchi@yahoo.it)

\begin{abstract}
Objective: Radiation therapy (RT) is a useful adjuvant tool for acromegalic patients not cured by surgery and/or not responding to pharmacotherapy. However, its specific effects on cardio- and cerebrovascular morbidity are still on debate.

Design: Retrospective analysis of 42 acromegalic patients cured after conventional radiotherapy (CRT, $n=31$ ) or radiosurgery by gamma-knife (GKRS, $n=11$ ) followed for a median period of 16.5 years (range: 2-40). Totally, 56 patients cured by surgery alone, with similar GH/IGF1 levels and duration of disease remission, served as control group.

Methods: Changes in cardiovascular risk factors, such as body mass index, glucose metabolism, insulin resistance, blood pressure, and lipid profile (pre-defined primary end point) and occurrence of new major cardio- and cerebrovascular events (secondary end point) during follow-up.

Results: The number of obese, hypertensive, and dyslipidemic subjects increased over time only in patients cured with RT. In contrast, the glucose response to the oral glucose tolerance test and the percentage of subjects with glucose alterations improved only in controls. As expected, the percentage of patients with pituitary failure was deeply higher among RT patients than among controls (86 vs $30 \%, P<0.0005)$. Despite these findings, a similar number of RT patients and controls developed major cardio- or cerebrovascular events (4/42 vs 3/56, P: NS). No differences were found between CRT and GKRS subgroups.

Conclusions: Previous RT seems to be associated with a worse metabolic profile in acromegalic patients studied after a long-term follow-up. Nevertheless, a direct link between RT and cardiovascular events remains to be proven.
\end{abstract}

European Journal of Endocrinology 164 675-684

\section{Introduction}

Acromegaly is an insidious disease caused by chronic GH and insulin-like growth factor 1 (IGF1) hypersecretion associated with increased morbidity and mortality mostly due to cardiovascular causes $(1,2)$. Cardiac diseases typically include a cardiomyopathy further deranged by some co-morbidities, which act as cardiovascular risk factors (i.e. abnormal glucose metabolism, insulin resistance, and arterial hypertension (1-3). Nevertheless, it is still unclear whether and to what extent acromegalic patients are at risk of coronary heart disease and atherosclerosis (4-6). Excess of hormone seems to be the main factor predictive for reduced life expectancy $(1,7,8)$. Thus, treatment of acromegaly aims to restore normal GH/IGF1 levels and to control both tumor growth and acromegaly-related complications in order to normalize quality of life and mortality, while trying to preserve anterior pituitary function (9). In this context, transsphenoidal surgery (TNS) and pharmacological therapy with long-acting somatostatin analogues (SSa) represent the most successful treatment options in clinical practice.

Radiation therapy (RT) is currently considered as a useful adjuvant tool mostly for difficult patients not successfully operated and/or inadequately responsive to SSa therapy $(10,11)$. Fractionated external megavoltage radiotherapy is only slowly effective on both tumor growth and GH hypersecretion and shows variable response rates. In recent studies, biochemical remission is reported in $30-80 \%$ of patients $10-20$ years after RT with a high incidence of secondary hypopituitarism (12-14). More recently, different stereotactic radiosurgery techniques, delivered in a single dose (i.e. gamma-knife, LINAC, Cyberknife, and proton beam), have been developed. In particular, gamma-knife 
radiosurgery (GKRS), the most investigated technique, has been reported to induce biochemical regression of acromegaly in $17-82 \%$ of cases in a short period of time with a variable frequency of pituitary failure (15-18). Nowadays, conventional radiotherapy (CRT) is usually performed in patients with diffuse and/or large tumor residuals, whereas GKRS is preferentially used for circumscript low-volume residuals localized far from the optic pathways (15).

It is well known that successful TNS is the only treatment able to reverse most acromegalic co-morbidities, including metabolic and cardiovascular diseases, normalizing life expectancy (19-21). In contrast, the long-lasting effects of chronic SSa treatment on cardiovascular mortality are still unknown, whereas those on glucose metabolism seem to be largely negative, even if this remains a matter of debate (21-23). RT has directly been associated with an increased mortality, mainly due to cerebrovascular causes, in patients with acromegaly and other pituitary disorders $(7,8,24,25)$. In addition, hypopituitarism, which is frequently induced by RT, has also been associated with increased mortality, predominantly for cardiovascular reasons (26-28). Thus, the definitive role of either RT per se or pituitary failure on the increased risk of cardio- and cerebro-vascular diseases in acromegaly has not been clearly demonstrated. Furthermore, the long-term influence of previous pituitary irradiation on single cardiovascular risk factors and metabolic parameters has been previously poorly investigated (12). Finally, the specific effects of GKRS on cardiovascular morbidity and mortality have not yet been investigated.

The predefined primary end point of this retrospective study was to compare the long-term effects of successful radiotherapy on glucose metabolism and other cardiovascular risk factors with those obtained after successful neurosurgery alone. Secondary end point was to evaluate the occurrence of major cardio- and cerebrovascular events during the follow-up.

\section{Materials and methods}

\section{Patients}

A total of 121 consecutive acromegalic patients (95 in Milan and 26 in Wuerzburg) underwent first-line or adjuvant radiant treatment between 1964 and 2009: 97 patients were treated with fractionated megavoltage external radiotherapy, in this study called CRT, and 24 with GKRS.

In order to avoid the possible influence of chronic GH/IGF1 hypersecretion and SSa administration, we selected only those patients who were cured for a minimum of 2 years while not receiving any pharmacological treatment for acromegaly (i.e. $n=62$ out of 121, 50 in Milan and 12 in Wuerzburg). Disease remission was established according to the absence of acromegaly-related symptoms and to the biochemical criteria valid at the time of the evaluation (post-glucose GH nadir $<1 \mu \mathrm{g} / \mathrm{l}$ and normal IGF1 $(29,30)$. Moreover, only patients with available hormonal and metabolic data both at the time of treatment (baseline) and after the long-term follow-up were considered suitable for the study. Thus, we excluded from the study 20 patients with too short follow-up (eight were lost at the follow-up, six had moved to another town, five were cured for $<2$ years, one had died because of acute myocardial infarction (AMI) 1 year after RT). Therefore, a total of 42 acromegalic patients (11 males and 31 females, age: $55 \pm 12$ years, 33 in Milan and nine in Wuerzburg), cured after CRT $(n=31)$ or GKRS $(n=11)$ and followed for a median period of 16.5 years (range: 2-40), were included in the present analysis. Most of them had previously undergone unsuccessful surgery $(n=39)$. In these cases, RT was performed after a median period of 12 months from TNS (range: 0-156).

A total of 56 acromegalic patients (24 males and 32 females, age: $57 \pm 11$ years, 46 in Milan and ten in Wuerzburg) treated with TNS alone and cured for $>2$ years were also investigated as control group.

Historical, clinical, and hormonal data from all the patients at baseline are summarized in Table 1 .

\section{Study protocol}

All the clinical, hormonal, and biochemical data recorded for each acromegalic patient at baseline, i.e. immediately before radiotherapy and after a long-term follow-up (at least 2 years after disease remission), were collected from patient's records. The same evaluation was performed for the control group. In particular, previous history of acromegaly and its treatment and the complete hormonal pattern $(\mathrm{GH}$, post-glucose $\mathrm{GH}$ nadir, IGF1, prolactin, basal thyroid, adrenal, and gonadic functions) were analyzed. Data from dynamic adrenal function assessed by the short Synacthen test (31) were included for all the patients. The stimulated $\mathrm{GH}$ secretion was assessed by arginine plus $\mathrm{GH}$ releasing hormone test (32) in 50 of 98 patients without any clinical pre-selection (28/42 RT patients and 22/56 controls, 47/79 from Milan and 3/19 from Wuerzburg). A series of metabolic parameters and cardiovascular risk factors, such as body mass index (BMI), fasting plasma glucose and fasting serum insulin (FG and FI), HbA1c, complete lipid profile (total cholesterol, lowdensity lipoprotein cholesterol, high-density lipoprotein cholesterol, and triglycerides), systolic and diastolic blood pressure, were also investigated. In addition, blood samples were collected every 30 min during a $2 \mathrm{~h} 75 \mathrm{~g}$ oral glucose tolerance test (OGTT), which was performed in most non-diabetic patients $(n=75)$, for measuring glucose and insulin levels. Fasting insulin resistance and secretion were calculated according to the homeostasis model assessment (HOMA) as follows: HOMA-IR =FI $(\mathrm{mU} / \mathrm{l}) \times \mathrm{FG}(\mathrm{mmol} / \mathrm{l}) / 22.5$ and 
Table 1 Clinical and hormonal characteristic of acromegalic patients treated with radiotherapy (RT group) or surgery alone (control group) at the time of the respective treatment (baseline).

\begin{tabular}{llll}
\hline & RT group $(n=42)$ & Control group $(n=56)$ & $P$ \\
\hline Sex (M/F) & $11 / 31$ & $24 / 32$ & 0.14 \\
Estimated disease duration & $4.8 \pm 3.7$ & $6.3 \pm 4.2$ & 0.11 \\
before diagnosis (years) & $39 \pm 13$ & $43 \pm 10$ & 0.06 \\
Age (years) & $39(93 \%)$ & $2(4 \%)$ & - \\
Previous neurosurgery, $n$ (\%) & $10.2 \pm 13.0$ & $17.2 \pm 14.2$ & $<\mathbf{0 . 0 5}$ \\
GH levels ( $\mu$ g/l) & $81 \pm 36$ & $108 \pm 43$ & $<\mathbf{0 . 0 5}$ \\
IGF1 levels (nmol/l) & $6.4 \pm 4.1$ & $10.0 \pm 5.6$ & $<\mathbf{0 . 0 5}$ \\
IGF1 levels (SDS) & $15(36 \%)$ & $5(9 \%)$ & $<0.05$ \\
Secondary hypopituitarism, $n(\%)$ & $26.5 \pm 3.3$ & $26.3 \pm 4.1$ & 0.85 \\
BMI (kg/m) & $90.3 \pm 7.8$ & $105.0 \pm 39.4$ & 0.07 \\
Fasting glucose levels (mg/dl) & $116.5 \pm 34.4$ & $122.6 \pm 37.5$ & 0.54 \\
Post-OGTT glucose levels (mg/dl) & $12.8 \pm 6.7$ & $13.8 \pm 5.7$ & 0.71 \\
Fasting insulin levels (mU/l) & $2.9 \pm 1.6$ & $3.4 \pm 1.6$ & 0.53 \\
HOMA-IR & $161.8 \pm 95.5$ & $160.4 \pm 73.9$ & 0.97 \\
HOMA-B\% & $122.1 \pm 13.0 / 79.3 \pm 9.3$ & $128.3 \pm 19.3 / 81.7 \pm 10.5$ & $0.13 / 0.31$ \\
SBP/DBP (mmHg) & $213 \pm 60$ & $210 \pm 54$ & 0.86 \\
Total cholesterol (mg/dl) & $158 \pm 138$ & $147 \pm 103$ & 0.83 \\
Triglycerides (mg/dl) & $45 \pm 8 / 134 \pm 51$ & $45 \pm 11 / 139 \pm 50$ & $0.99 / 0.82$ \\
HDL/LDL cholesterol (mg/dl) & & & \\
\hline
\end{tabular}

M, males; F, females; BMI, body mass index; OGTT, $2 \mathrm{~h}$ oral glucose tolerance test; HOMA-IR=FI $(\mathrm{mU} / \mathrm{l}) \times \mathrm{FG}(\mathrm{mmol} / \mathrm{l}) / 22.5$ and HOMA-B\% $=((\mathrm{FI}(\mathrm{mU} / \mathrm{l}) \times 20) /(\mathrm{FG}(\mathrm{mmol} / \mathrm{l})-3.5))$ (Matthews 1985) (33); SBP, systolic blood pressure; DBP, diastolic blood pressure; $\mathrm{HDL}$, high-density lipoprotein; LDL, low-density lipoprotein. To convert IGF1 concentrations to nmol/l divide concentration in $\mu \mathrm{g} / \mathrm{l}$ by 7.741 . Significant values are in boldface.

HOMA-B $\%=(($ FI $\quad(\mathrm{mU} / \mathrm{l}) \times 20) /(\mathrm{FG} \quad(\mathrm{mmol} / \mathrm{l})-3.5))$ respectively (33).

Patients were considered affected by arterial hypertension and/or dyslipidemia when treated with antihypertensive or antihyperlipidemic drugs or according to the ATPIII criteria (34). Diabetes mellitus and glucose tolerance alterations were defined according to the newly proposed cutoff values (35). Different antidiabetic treatments were also considered.

Previous family and personal history for ischemic cardiovascular disease and current smoking status were also investigated. Family history was regarded as positive if first-grade relatives had a major cardio- or cerebrovascular event before the age of 55 years in males or 65 years in females.

Furthermore, the incidence of new cardio- (i.e. angina or AMI) or cerebrovascular events (i.e. transitory ischemic attack (TIA), and stroke) after radiotherapy or surgery was investigated based on the patient certified charts and by a series of instrumental evaluation such as cardiac echo-color Doppler ultrasound, basal and dynamic ECG, and cerebral magnetic resonance imaging as appropriate. Evidence of chronic coronary heart disease or cerebral vasculopathy was also registered. The entire study protocol was approved by both local ethical committees.

\section{Procedures and assays}

Concerning the megavoltage CRT, the median dose delivered was $50 \mathrm{~Gy}$ (range: 43-55), fractionated in 30-35 sessions $(1.5 \pm 1.8$ Gy for each application).
Patients (70\%) were irradiated with a three-field technique, using a ${ }^{60}$ Co source (13), whereas no information could be obtained on the technique used for the remaining patients who had been treated in other centers. The GKRS treatment was performed only in patients followed in Milan, the median dose being 45 Gy (range: 30-80), the median margin dose $21 \mathrm{~Gy}$ (range: 15-35), and the median percentage isodose 50 (range: 45-60). A detailed description of GKRS has been reported previously $(15,18)$.

Serum GH levels were assessed in Milan utilizing a highly sensitive two-site monoclonal immunofluorometric assay (AutoDelfia kit, Wallac, Inc. OY, Turku, Finland), the standards being calibrated against the first WHO IRP 80/505. In Wuerzburg, the GH was measured utilizing a chemiluminescence method (DPC Immulite 2000, Los Angeles, CA, USA), the standards being calibrated against the WHO NIBSC 2nd IS $95 / 574$. The detection limit of both assays was $0.01 \mu \mathrm{g} / \mathrm{l}$. Serum IGF1 concentrations were assessed in Milan by the commercial radioimmunometric assay kit of Mediagnost (Tübingen, Germany) until 2008. The intra- and inter-assay coefficients of variation (CV) were 3.2 and $8.9 \%$ respectively. Afterwards and in Wuerzburg, the chemiluminescence method by DPC Immulite 2000 was used (intra- and inter-assay CV below $8 \%$ ). The IGF1 values were compared to the appropriate sex- and age-adjusted range and expressed as SDS. Serum cortisol, TSH, free thyroxine, and prolactin levels were determined by an immunofluorometric assay (AutoDelfia kit). All the other hormonal, biochemical, and metabolic parameters were measured by standard procedures. 


\section{Statistical analysis}

The results are expressed as mean \pm s.D. The area under the response curve (AUC) of glucose and insulin during the OGTT were estimated according to the trapezoidal method. The differences between baseline and the last visit was calculated as follows: delta percentage $(\Delta \%)=$ $100 \times$ (baseline value -last value)/baseline value. A paired or unpaired Student's t-test was performed to compare different variables when data were normally distributed. Otherwise, the non-parametric Wilcoxon or Mann-Whitney $U$ tests were used. A Fisher's exact test or a $\chi^{2}$ test, as appropriate, was used to compare categorical data. Correlations between different parameters were evaluated by linear regression analysis for continuous variables or logistic regression analysis for dichotomic variables. Differences between curves were evaluated by a regular two-way ANOVA followed by the Bonferroni post hoc test. Longitudinal evaluations were performed by Kaplan-Meier method, and differences between subgroups were evaluated by log-rank test. The hazard ratio (HR) and the $95 \%$ confidence interval (95\% CI) of ratio were reported. Factors associated with major ischemic events at a significance level of $<0.10$ were entered into a stepwise forward analysis to establish the rank of predictors (multivariate Cox regression analysis). All statistical examinations were performed using GraphPad Prism (Version 4.0; San Diego, CA, USA) or SPSS (Version 11.0, SPSS, Inc., Chicago, IL, USA). Values of $P<0.05$ were considered statistically significant.

\section{Results \\ Hormonal data (GH/IGF1 secretion and pituitary functions)}

The median period of follow-up in the RT was 16.5 years (range: 2-40), being slightly longer than that in the

Table 2 Clinical and hormonal characteristic of cured acromegalic patients treated with radiotherapy (RT group) or surgery alone (control group) at the last visit after a long-term follow-up.

\begin{tabular}{|c|c|c|c|}
\hline & RT group $(n=42)$ & Control group $(n=56)$ & $\boldsymbol{P}$ \\
\hline Age (years) & $55 \pm 12$ & $57 \pm 11$ & 0.49 \\
\hline Time of overall follow-up (months) & $205 \pm 104$ & $162 \pm 84$ & $<0.05$ \\
\hline Duration of active disease (months) ${ }^{a}$ & $163 \pm 106$ & $76 \pm 50$ & $<0.0005$ \\
\hline Time since disease remission (months) & $128 \pm 99$ & $161 \pm 83$ & 0.08 \\
\hline \multicolumn{4}{|l|}{ Hormonal pattern } \\
\hline GH levels $(\mu \mathrm{g} / \mathrm{l})$ & $0.85 \pm 0.89$ & $0.89 \pm 1.3$ & 0.86 \\
\hline IGF1 levels (nmol/l) & $18.6 \pm 7.6$ & $18.8 \pm 7.7$ & 0.90 \\
\hline IGF1 levels (SDS) & $-0.8 \pm 1.1$ & $-0.9 \pm 1.0$ & 0.77 \\
\hline Secondary hypopituitarism, $n(\%)$ & $36(86 \%)$ & $17(30 \%)$ & $<0.0005$ \\
\hline Multiple failures, $n(\%)$ & $24(57 \%)$ & $6(11 \%)$ & $<0.0005$ \\
\hline Hypoadrenalism, $n(\%)$ & $22(52 \%)$ & $6(11 \%)$ & $<0.0005$ \\
\hline Hypogonadism, $n(\%)$ - males/females & $19(45 \%)-8 / 11$ & $9(16 \%)-4 / 5$ & $<0.005$ \\
\hline Hypothyroidism, $n(\%)$ & $20(48 \%)$ & $2(4 \%)$ & $<0.0005$ \\
\hline Total GH deficiency, $n(\%)^{\mathrm{b}}$ & $17 / 28(61 \%)$ & $9 / 22(41 \%)$ & $<0.05$ \\
\hline \multicolumn{4}{|l|}{ Cardiovascular risk factors } \\
\hline Family history for ischemic diseases, $n(\%)$ & $8(19 \%)$ & $5(9 \%)$ & 0.23 \\
\hline Smokers, $n(\%)$ & $12(29 \%)$ & $25(45 \%)$ & 0.16 \\
\hline Obesity, $n(\%)$ & $17(40 \%)$ & $16(29 \%)$ & 0.38 \\
\hline BMl $\left(\mathrm{kg} / \mathrm{m}^{2}\right)$ & $29.3 \pm 5.1$ & $28.0 \pm 5.4$ & 0.25 \\
\hline Abnormal glucose metabolism (DM/IGT/N) & $3 / 8 / 31$ & $7 / 4 / 4 \overline{5}$ & 0.17 \\
\hline Fasting glucose levels (mg/dl) & $89.8 \pm 13.5$ & $95.3 \pm 25.2$ & 0.23 \\
\hline Post-OGTT glucose AUC (g/dl per $120 \mathrm{~min}$ ) & $16.6 \pm 4.9$ & $14.4 \pm 4.0$ & 0.06 \\
\hline HOMA-IR & $2.0 \pm 1.6$ & $1.9 \pm 1.5$ & 0.82 \\
\hline HOMA-B\% & $118.5 \pm 110.7$ & $131.4 \pm 129.6$ & 0.64 \\
\hline $\mathrm{HbA1c}(\%)$ & $5.7 \pm 0.5$ & $5.9 \pm 1.2$ & 0.47 \\
\hline Blood hypertension, $n(\%)^{d}$ & $22(52 \%)$ & $25(45 \%)$ & 0.45 \\
\hline SBP/DBP $(\mathrm{mmHg})$ & $128.7 \pm 17.5 / 82.6 \pm 10.7$ & $126.0 \pm 15.1 / 78.5 \pm 13.6$ & $0.32 / 0.09$ \\
\hline Dyslipidemia, $n(\%)^{d}$ & $27(64 \%)$ & $28(50 \%)$ & 0.16 \\
\hline Triglycerides (mg/dl) & $147 \pm 67$ & $119 \pm 74$ & 0.14 \\
\hline HDL cholesterol $(\mathrm{mg} / \mathrm{dl})$ & $52 \pm 12$ & $53 \pm 16$ & 0.75 \\
\hline LDL cholesterol (mg/dl) & $135 \pm 41$ & $140 \pm 33$ & 0.49 \\
\hline
\end{tabular}

BMI, body mass index; DM, diabetes mellitus; IGT, impaired glucose tolerance; OGTT, 2 h oral glucose tolerance test; AUC, area under the curve, HOMA-IR= $\mathrm{FI}(\mathrm{mU} / \mathrm{l}) \times \mathrm{FG}(\mathrm{mmol} / \mathrm{l}) / 22.5$ and HOMA-B\% $=((\mathrm{FI}(\mathrm{mU} / \mathrm{l}) \times 20) /(\mathrm{FG}(\mathrm{mmol} / \mathrm{l})-3.5))$ (Matthews 1985) (33); SBP, systolic blood pressure; DBP, diastolic blood pressure; HDL, high-density lipoprotein; LDL, low-density lipoprotein. To convert IGF1 concentrations to nmol/l, divide concentration in $\mu \mathrm{g} / \mathrm{l}$ by 7.741 .

Significant $P$ values are in boldface.

a Duration of active disease $=$ estimated time of active disease before diagnosis + time between diagnosis and treatment + time of active disease after treatment.

${ }^{\mathrm{b}}$ Percentage of the patients tested with the arginine $+\mathrm{GHRH}$ stimulation test.

${ }^{\mathrm{C}} \mathrm{DM}$ and IGT judged according to the new criteria (Jermendy 2004) (35).

dJudged according to the ATPIII criteria (2001) (34). 
control group (13 years, range: $2-30, P<0.05)$. The entire duration of active acromegaly disease was also longer in the RT group (Table 2). However, most importantly, the period elapsed from the time of treatment till the documented remission of acromegaly was not different between the two groups (Table 2).

Even if the mean GH and IGF1 levels recorded at baseline were higher in controls, all of which were newly diagnosed acromegalics, than in RT patients (Table 1), at the last follow-up visit, they were superimposable between the two groups (Table 2). In particular, IGF1 levels were below the normal range in a similar proportion of patients (14\% of RT and $11 \%$ of control patients, $P$ : NS). The IGF1 SDS values negatively correlated only in the RT group with the duration of the entire follow-up $(P<0.05, r=0.30)$ and with the BMI values $(P<0.01, r=0.44)$. The post-glucose $\mathrm{GH}$ nadir levels were higher in RT patients than in controls $(0.37 \pm 0.23$ vs $0.23 \pm 0.26 \mu \mathrm{g} / \mathrm{l}, P<0.05)$, and a lower, but not significantly, number of RT patients had a GH nadir below the new cutoff of $0.4 \mu \mathrm{g} / \mathrm{l}$ (36) with respect to the controls (59 vs $80 \%, P=0.06$ ).

As expected, the number of patients with one or more anterior-pituitary failures was always higher in the RT than in the control group ( 86 vs $30 \%, P<0.0005$ ). GH deficiency was also more frequent among the RT patients (Table 2). The hormonal insufficiencies observed at the last visit are shown in detail in Table 2. All the pituitary deficits were adequately substituted with appropriate hormonal replacement therapy. In particular, patients with hypoadrenalism received either cortone acetate per os $(n=18$, median dose $25 \mathrm{mg} / \mathrm{die})$ or hydrocortisone per os $(n=10$, median dose $=15 \mathrm{mg} /$ die, range: $10-30$ ). Only ten hypogonadic females, three hypogonadic males, and 21 GHD patients $(13 / 17$ in the RT and 8/9 in the TNS group, $P$ NS) were not treated with replacement treatment due to recent discovery of the insufficiencies, advanced age, or patient's refusal.

\section{Cardiovascular risk factors (BMI, glucose metabolism, insulin resistance, blood pressure, and lipid profile)}

The number of patients with a positive family or personal previous history of ischemic disease and the number of smokers were similar between the RT and the control groups (Table 2).

At baseline, RT patients and controls were comparable in terms of BMI, glucose levels, insulin resistance, blood pressure, and lipid profile (Table 1 and Fig. 1). During the follow-up, the mean BMI values significantly increased in both the groups (Tables 1 and 2 , both $P<0.005)$. In spite of this, FI levels slightly decreased, though not significantly, over time in both the RT and the control groups (from $12.8 \pm 6.7$ to 8.5 \pm 6.2 and from $13.8 \pm 6.7$ to $7.9 \pm 5.0 \mathrm{mUI} / \mathrm{l}$ respectively, both $P=0.08$ ). On the other hand, no significant changes of either fasting or post-2 h OGTT glucose or $\mathrm{HbA1c}$ levels were found in both the groups. Nevertheless, the post-OGTT glucose AUC levels (AUCglu) decreased only in patients treated with surgery alone (from $16.8 \pm 3.6$ to $14.4 \pm 4.0 \mathrm{~g} / \mathrm{dl}$ per $120 \mathrm{~min}$, $P=0.05$ versus baseline and $P=0.06$ versus the RT group). The AUCglu $\Delta \%$ with respect to baseline was also significantly different between RT patients and controls $(+10.4 \pm 35 \%$ vs $-9.5 \pm 24 \%, \quad P<0.05)$. These last findings might be somehow influenced by a worse baseline glucose tolerance observed in the control group (Fig. 1); however, the trend of the glucose curve after OGTT evolved clearly differently over time in the two groups, becoming significantly higher at the last visit in the RT group $(P<0.005$ by two-way ANOVA, Fig. 2). No other significant changes in the evaluated cardiovascular risk factors and metabolic parameters were observed over the time (Table 2), including the need of anti-diabetic, anti-hypertensive, and antidyslipidemic therapies.

Considering the percentages of patients affected by different metabolic alterations, the number of subjects with different glucose tolerance alterations (diabetes mellitus, impaired glucose tolerance (IGT), and IFG) decreased during the follow-up in the control group (from 37 to $20 \%, P=0.05$ ), whereas slightly increased in the RT group (from 14 to $26 \%, P: N S$ ). On the other hand, the proportion of overweight, hypertensive, and
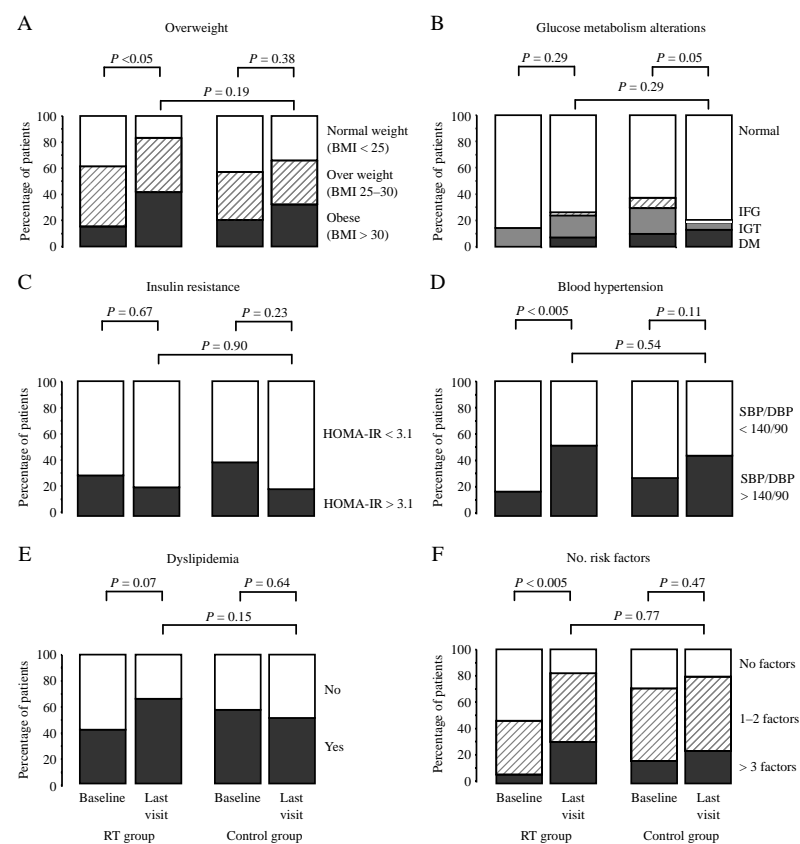

Figure 1 Percentage of patients affected by overweight (A), insulin resistance evaluated by HOMA-IR (B), glucose metabolism alterations $(C)$, blood hypertension (D), dyslipidemia $(E)$, and by different cardiovascular risk factors $(F)$ in cured acromegalic patients treated with radiotherapy (RT group, $n=42$ ) or surgery alone (control group, $n=56$ ) before the corresponding treatment (baseline) and at the last visit after a long-term follow-up. 

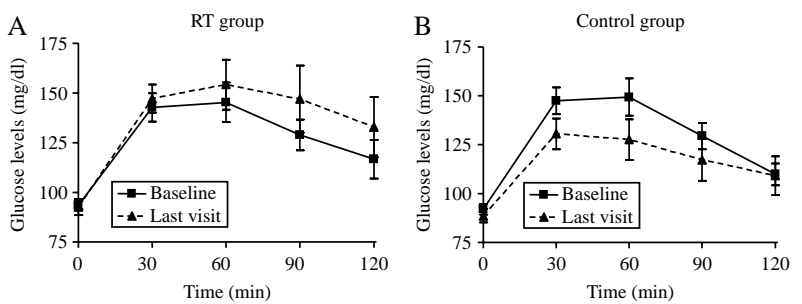

Figure 2 Glucose levels during oral glucose tolerance test in cured acromegalic patients treated with radiotherapy (RT group, A) or surgery alone (control group, B) before the corresponding treatment (baseline) and at the last visit after a long-term follow-up (both $P N S$ versus baseline by two-way ANOVA for repeated measures). The entire curve recorded at the last visit was significantly different between the two treatment groups $(P<0.005$ by two-way ANOVA).

dyslipidemic subjects increased among the RT patients (Fig. 1). Therefore, the number of patients with at least one cardiovascular risk factor significantly rose over the time in the RT group (Fig. 1). This trend was not associated with either the presence of pituitary failures, including hypoadrenalism and/or GHD per se, or with any hormonal replacement therapy.

\section{Comparison between CRT and GKRS}

The length of the entire follow-up and of the time passed since the definitive remission of acromegaly was longer in patients treated with CRT than in those treated with GKRS (19 vs 11 years and 7.5 vs 5 years respectively, both $P<0.05)$. Nevertheless, the time passed between radiotherapy and disease remission and thus the entire duration of active disease were similar between the two subgroups (Table 3). Basal GH levels were lower in the fractionated external RT than in the GKRS subgroup, but both the GH nadir levels and the IGF1 SDS were similar (Table 3). The number of patients with one or more anterior-pituitary failures at the last follow-up visit was higher in the CRT than in the GKRS subgroup (97 vs 55\%, $P<0.005$, Table 3), the most important differences being the frequency of multiple deficiencies ( 74 vs $9 \%, P<0.0005$ ) and secondary hypoadrenalism (68 vs $9 \%, P<0.005$ ). The occurrence of new pituitary deficiencies was higher among patients treated with CRT, even adjusting it for the entire duration of follow-up.

Despite all these disparities, no significant differences were observed in the changes of metabolic parameters and cardiovascular risk factors between the two different RT subgroups, except for triglycerides levels (Table 3).

\section{Cardio- and cerebrovascular morbidity (acute events and chronic diseases)}

A total of four patients in the RT group (three males and one female) and three patients in the control group (three males) developed a major cardio- or cerebrovascular event during the entire follow-up (9.5 vs $5.3 \%$ of total, P: NS). In particular, two patients in the CRT subgroup developed a coronary heart disease (2 AMI, $4.7 \%$ of RT patients, $6.4 \%$ of CRT patients, $P$ : NS versus GKRS subgroup and control group). On the other hand, five patients had a cerebrovascular accident. Two were in the GKRS subgroup (2 TIA, $4.7 \%$ of RT, $18 \%$ of GKRS, $P=0.063$ versus CRT) and three in the control group (2 TIA and 1 stroke, 5.3\% of controls, P: NS versus RT). None of these events was fatal. The overall median period elapsed from the time of treatment was 78 months (range: 24-300) in the RT group and 48 months (range: $24-60)$ in the control group $(P=0.37)$.

At the Kaplan-Meier analysis, only the male gender resulted highly associated with the development of a major cardiovascular event $(P<0.005, \mathrm{HR}=15.0,95 \%$ $\mathrm{CI}=3.0-60.4)$. Other known risk factors such as hypertension $(P=0.12, \mathrm{HR}=3.2,95 \% \mathrm{CI}=0.7-12.2)$ and advanced age $(P=0.29, \mathrm{HR}=2.0,95 \% \mathrm{CI}=0.5-9.7)$ were only slightly associated with the risk of cardio- or cerebrovascular events. On the contrary, the duration of the follow-up, previous RT (including subdivided CRT and GKRS), secondary hypopituitarism (including secondary hypoadrenalism by itself), replacement therapy, post-OGTT GH nadir levels, IGF1 SDS, obesity, and glucose metabolism or lipid profile alterations resulted as not predictive. In the multivariate analysis, including gender, age, and presence of hypertension besides type of treatment and pituitary deficits, only male gender and age were the significant predictive factors. No factor remains significant when the analysis was corrected either for the duration of the follow-up or for the period passed from disease remission.

Another female patient in the control group showed evidence for chronic coronary (i.e. anterior interventricular trunk) occlusion. Including her in the univariate analysis (log-rank test), the results did not substantially change. In addition, one female patient treated with CRT died from pulmonary venous thromboembolism at the age of 74 years, after 27 years from treatment. She was also suffering from arterial hypertension, IGT, and multiple pituitary failures.

Finally, two patients, who previously underwent CRT, developed a meningioma after 23 and 22 years from therapy ( $P=0.18$ versus control group).

\section{Discussion}

In this study, we demonstrate that the RT is not able to revert most of the acromegaly-related cardiovascular risk factors, even when it is successful from an endocrine perspective. In previous papers, we already evaluated the efficacy of RT, both as fractionated external CRT and as GKRS, on biochemical remission and on tumor growth $(13,15,18)$. In this study, we strictly focus on the effects of RT on several risk factors, which may be partly responsible for the increased 
Table 3 Hormonal and metabolic characteristic of acromegalic patients cured after CRT or GKRS after a long-term follow-up.

\begin{tabular}{|c|c|c|c|}
\hline & CRT group $(n=31)$ & GKRS group $(n=11)$ & $P$ \\
\hline Age (years) & $55.6 \pm 12.1$ & $54.5 \pm 12.7$ & 0.80 \\
\hline Time of overall follow-up (months) & $231 \pm 109$ & $133 \pm 33$ & $<0.05$ \\
\hline Time from RT to remission (months) & $96 \pm 103$ & $68 \pm 33$ & 0.41 \\
\hline Time since disease remission (months) & $149 \pm 105$ & $65 \pm 31$ & $<0.05$ \\
\hline GH levels $(\mu \mathrm{g} / \mathrm{l})$ & $0.6 \pm 0.5$ & $1.5 \pm 1.3$ & $<0.005$ \\
\hline Post-OGTT GH nadir levels $(\mu \mathrm{g} / \mathrm{l})$ & $0.33 \pm 0.25$ & $0.44 \pm 0.17$ & 0.26 \\
\hline IGF1 levels (nmol/l) & $17.2 \pm 7.1$ & $22.5 \pm 8.0$ & $<0.05$ \\
\hline IGF1 levels (SDS) & $-1.0 \pm 1.1$ & $-0.4 \pm 0.9$ & 0.11 \\
\hline Secondary hypopituitarism, $n(\%)$ & $30(97 \%)$ & $6(54 \%)$ & $<0.005$ \\
\hline Multiple failures, $n(\%)$ & $23(74 \%)$ & $1(9 \%)$ & $<0.0005$ \\
\hline Hypoadrenalism, $n(\%)$ & $21(68 \%)$ & $1(9 \%)$ & $<0.005$ \\
\hline Hypogonadism, $n(\%)-$ males/females & $16(52 \%)-5 / 11$ & $3(27 \%)-3 / 0$ & NS \\
\hline Hypothyroidism, $n(\%)$ & $18(58 \%)$ & $2(18 \%)$ & $<0.05$ \\
\hline Total GH deficiency, $n(\%)$ & $16 / 20(80 \%)$ & $1 / 8(12.5 \%)$ & $<0.05$ \\
\hline Obesity, $n(\%)$ & $14(45 \%)$ & $3(27 \%)$ & 0.30 \\
\hline BMI $\left(\mathrm{kg} / \mathrm{m}^{2}\right)$ & $29.2 \pm 5.4$ & $29.5 \pm 4.6$ & 0.88 \\
\hline $\begin{array}{l}\text { Abnormal glucose metabolism } \\
\text { (DM/IGT/normal glucose tolerance) }^{a}\end{array}$ & $3 / 5 / 23$ & $0 / 2 / 9$ & 0.56 \\
\hline Fasting glucose levels (mg/dl) & $88.1 \pm 14.4$ & $94.5 \pm 9.6$ & 0.18 \\
\hline Post-OGTT glucose levels (mg/dl) & $120 . \overline{3} \pm 53.1$ & $137 . \overline{9} \pm 65.5$ & 0.44 \\
\hline Fasting insulin levels (mU/l) & $8.1 \pm 5.9$ & $9.1 \pm 6.7$ & 0.69 \\
\hline HOMA-IR & $1.9 \pm 1.7$ & $2.1 \pm 1.6$ & 0.72 \\
\hline HOMA-B\% & $123.4 \pm 119.3$ & $110.8 \pm 101.1$ & 0.77 \\
\hline $\mathrm{HbA1c}(\%)$ & $5.8 \pm 0.5$ & $5.7 \pm 0.5$ & 0.50 \\
\hline Blood hypertension, $n(\%)^{\mathrm{b}}$ & $16(52 \%)$ & $4(36 \%)$ & 0.38 \\
\hline SBP/DBP $(\mathrm{mmHg})$ & $130.0 \pm 18.6 / 83.1 \pm 12.0$ & $125.5 \pm 15.1 / 81.4 \pm 7.1$ & $0.44 / 0.66$ \\
\hline Dyslipidemia, $n(\%)^{\mathrm{b}}$ & $21(68 \%)$ & $6(54 \%)$ & 0.43 \\
\hline Total cholesterol (mg/dl) & $218.5 \pm 42.0$ & $213.8 \pm 49.7$ & 0.77 \\
\hline Triglycerides $(\mathrm{mg} / \mathrm{dl})$ & $157.6 \pm 66.8$ & $103.7 \pm 52.8$ & $<0.05$ \\
\hline HDL/LDL cholesterol (mg/dl) & $52.4 \pm 12.9 / 132.2 \pm 41.7$ & $51.4 \pm 8.6 / 141.7 \pm 40.4$ & $0.81 / 0.53$ \\
\hline
\end{tabular}

$\mathrm{BMI}$, body mass index; DM, diabetes mellitus; IGT, impaired glucose tolerance; OGTT, 2 h oral glucose tolerance test; $\mathrm{HOMA}-\mathrm{IR}=\mathrm{FI}(\mathrm{mU} / \mathrm{I}) \times \mathrm{FG}$ $(\mathrm{mmol} / \mathrm{l}) / 22.5$ and HOMA-B\% $=((\mathrm{FI}(\mathrm{mU} / \mathrm{l}) \times 20) /(\mathrm{FG}(\mathrm{mmol} / \mathrm{l})-3.5))$ (Matthews 1985) (33); SBP, systolic blood pressure; DBP, diastolic blood pressure; HDL, high-density lipoprotein; LDL, low-density lipoprotein. To convert IGF1 concentrations to nmol/l, divide concentration in $\mu \mathrm{g} / \mathrm{l}$ by 7.741 .

Significant $P$ values are in boldface.

aDM and IGT judged according to the new criteria (Jermendy 2004) (35).

bJudged according to the ATPIII criteria (2001) (34).

mortality in acromegaly $(2,7,8)$. The inclusion criteria and the study protocol were carefully designed in order to exclude possible confounding factors and biases. In particular, to avoid the influence of GH/IGF1 hypersecretion and to leave out the known metabolic effects of SSa administration (21), we studied only patients cured by surgery plus RT without concomitant pharmacotherapy, comparing them with patients treated with surgery alone. As expected, the number of subjects with anterior pituitary insufficiencies was much higher among subjects treated with RT $(18,34)$. As far as the metabolic and cardiovascular parameters are concerned, an improvement in the glucose response to OGTT was observed only in the control group. In addition, the number of patients with altered glucose tolerance decreased over time in controls only, while the number of obese, dyslipidemic, and hypertensive subjects increased in the RT group (even if they were similar in the two groups at the end of follow-up). These findings might be explained by either the higher prevalence of pituitary deficiencies, which also correlated with the AUCglu values, or the respective hormonal replacement therapies in the RT group.
However, also due to a relatively small number of patients, we did not find a direct relationship in the presence of hypopituitarism (including hypoadrenalism and GHD) on cardiovascular risk factors. Another possible explanation might be the longer time of follow-up and the longer duration of active acromegaly before remission in the RT group. In addition, with regard to hypertension, a possible direct role of RT on hypothalamic centers responsible for blood pressure control could be hypothesized. Moreover, the alterations in glucose homeostasis might reflect a different $\mathrm{GH}$ secretion pattern after RT and TNS. The latter hypothesis has been suggested by previous studies (37) and might be consistent with the higher postOGTT GH nadir values observed in our RT series despite a higher proportion of GHD patients. Thus, the longterm effects of RT, which is often and inevitably associated with pituitary failures and a long previous duration of active disease, on cardiovascular risk factors seem to be not fully comparable to those obtained with surgery alone. Rather, surgery remains the only treatment with a proven positive effect on overall metabolic alterations (19-21). 
A similar occurrence of major cardio- or cerebrovascular events was observed in the RT and the control patients (9.5 vs 5.3\%). Most were cerebrovascular incidents (five out of seven events) and none was fatal. The only predictive factor in our series was male gender, whereas age and hypertension played a secondary role. Previous reports described an increased risk of overall and cerebrovascular mortality after pituitary irradiation and/or in hypopituitarism (24-28), including central hypoadrenalism per se and hydrocortisone replacement therapy $(25,38)$. Interestingly, in this study, neither previous RT nor hypopituitarism nor replacement therapies (including adrenal insufficiency and glucocorticoid replacement therapy) were relevant factors predictive for cardiovascular events. It is likely that again the relatively small number of patients did preclude the chance to observe an increase in mortality/morbidity after RT due to the rarity of these events. Nevertheless, several further hypotheses might be proposed to explain the differences with previous reports. First of all, we directly compared only acromegalic patients cured according to the criteria valid at the time of the evaluation (thus including the post-glucose GH nadir, 26, 27), while off pharmacological treatment. In fact, even if it has been recently shown that probably acromegaly does not directly increase the risk for coronary artery disease (6), a role of GH/IGF1 excess on cardio- and cerebro-vascular morbidity/mortality has also been suggested $(7,8)$. In contrast, most of the previous works considered either different pituitary adenomas $(24,27)$ or a mixed series of controlled and not controlled acromegalic patients $(7,8,24,25)$, defining the disease remission by nonspecified criteria $(7,24,25)$ or by basal GH levels alone (8). Moreover, concerning the impact of glucocorticoid replacement therapy, our patients were treated with lower doses of hydrocortisone than those reported by Sherlock et al. (25) and a different corticosteroid (cortone acetate) was used in most of the patients. Finally, the progressive increase in the number of hypertensive, obese, and dyslipidemic patients observed in the RT group might eventually lead also to an increased risk of cardio- and cerebro-vascular events, which we were not able to detect due to the rather small number of patients. In conclusion, larger epidemiological studies are needed to clarify the impact of both previous RT and secondary hypopituitarism on cardioand cerebrovascular morbidity in acromegaly. Considering that chronic SSa administration has been shown to impair glucose metabolism (21), another study comparing the long-term effects of RT and SSa would also be of major interest.

The two different RT methods, namely CRT and GKRS, were also compared. It is currently recognized that GKRS has an efficacy on hormonal secretion similar to that of CRT, even if it requires a slightly shorter period to induce disease remission $(10,39,40)$ and usually causes fewer pituitary failures. Moreover, so far, an increased mortality from cerebrovascular disease has only been reported after treatment with CRT $(7,8$, $24,25)$. Our results demonstrated that, despite a longer period passed from acromegaly remission, lower GH and IGF1 levels, and a higher amount of pituitary failures (particularly of ACTH deficiency) among patients treated with CRT, the long-term effects of both the evaluated RT techniques on cardiovascular risk factors and on the cardio- or cerebro-vascular events were similar. However, we could hypothesize that the significantly lower IGF1 SDS in the CRT subgroup, which was the most numerous, could be co-responsible of a higher glycemic derangement. Finally, a relatively higher risk of developing iatrogenic meningioma was observed only after CRT. Again, the number of patients treated with GKRS was too small to draw firm conclusions.

In summary, both CRT and the GKRS are not able to fully reverse all the cardiovascular risk factors, despite inducing a complete biochemical remission of acromegaly. This holds true in particular for alterations of glucose metabolism. Thus, careful monitoring of glucose tolerance is advised in patients after RT. However, we found no evidence that radiotherapy for acromegaly is directly linked per se to an increased risk of cardio- or cerebro-vascular events, although larger epidemiological studies will be needed to fully elucidate the effects of RT on cardiovascular outcome.

Our findings may contribute to the current debate on the management of acromegaly $(41,42)$, confirming again that surgery remains the gold standard treatment. Nonetheless, RT currently remains useful as a salvage therapy for acromegalic patients not cured by surgery and/or not responding to pharmacotherapy.

\section{Declaration of interest}

The authors declare that there is no conflict of interest that could be perceived as prejudicing the impartiality of the research reported.

\section{Funding}

This study was partially supported by 'L'Oréal Italia per le Donne e per la Scienza' (C L Ronchi) and by Ricerca Corrente, Fondazione IRCCS Ospedale Maggiore Policlinico, Milan, Italy.

\section{Acknowledgements}

The authors are grateful to all the nursing staff and technical assistants for their expert and kind help.

\section{References}

1 Holdaway IM, Rajasoorya RC \& Gamble GD. Factors influencing mortality in acromegaly. Journal of Clinical Endocrinology and Metabolism 2004 89 667-674. (doi:10.1210/jc.2003-031199) 
2 Dekkers OM, Biermasz NR, Pereira AM, Romijn JA \& Vandenbroucke JP. Mortality in acromegaly: a metaanalysis. Journal of Clinical Endocrinology and Metabolism 200893 61-67. (doi:10.1210/jc.2007-1191)

3 Berg C, Petersenn S, Lahner H, Herrmann BL, Buchfelder M, Droste M, Stalla GK, Strasburger CJ, Roggenbuck U, Lehmann N, Moebus S, Jöckel KH, Möhlenkamp S, Erbel R, Saller B, Mann K \& Investigative Group of the Heinz Nixdorf Recall Study and the German Pegvisomant Observational Study Board and Investigators. Cardiovascular risk factors in patients with uncontrolled and long-term acromegaly: comparison with matched data from the general population and the effect of disease control. Journal of Clinical Endocrinology and Metabolism 201095 3648-3656. (doi:10.1210/jc.2009-2570)

4 Cannavo S, Almoto B, Cavalli G, Squadrito S, Romanello G, Vigo MT, Fiumara F, Benvenga S \& Trimarchi F. Acromegaly and coronary disease: an integrated evaluation of conventional coronary risk factors and coronary calcifications detected by computed tomography. Journal of Clinical Endocrinology and Metabolism 200691 3766-3772. (doi:10.1210/jc.2005-2857)

5 Bogazzi F, Battolla L, Spinelli C, Rossi G, Gavioli S, Di Bello V, Cosci C, Sardella C, Volterrani D, Talini E, Pepe P, Falaschi F, Mariani G \& Martino E. Risk factors for development of coronary heart disease in patients with acromegaly: a five-year prospective study. Journal of Clinical Endocrinology and Metabolism 200792 4271-4277. (doi:10.1210/jc.2007-1213)

6 Akutsu H, Kreutzer J, Wasmeier G, Ropers D, Rost C, Möhlig M, Wallaschofski H, Buchfelder M \& Schöfl C. Acromegaly per se does not increase the risk for coronary artery disease. European Journal of Endocrinology 2010162 879-886. (doi:10.1530/EJE09-0945)

7 Ayuk J, Clayton RN, Holder G, Sheppard MC, Stewart PM \& Bates AS. Growth hormone and pituitary radiotherapy, but not serum insulin-like growth factor-I concentrations, predict excess mortality in patients with acromegaly. Journal of Clinical Endocrinology and Metabolism 200489 1613-1617. (doi:10. 1210/jc.2003-031584)

8 Kauppinen-Mäkelin R, Sane T, Reunanen A, Välimäki MJ, Niskanen L, Markkanen H, Löyttyniemi E, Ebeling T, Jaatinen P, Laine H, Nuutila P, Salmela P, Salmi J, Stenman UH, Viikari J \& Voutilainen E. A nationwide survey of mortality in acromegaly. Journal of Clinical Endocrinology and Metabolism $2005 \mathbf{9 0}$ 4081-4086. (doi:10.1210/jc.2004-1381)

9 Melmed S, Colao A, Barkan A, Molitch M, Grossman AB, Kleinberg D, Clemmons D, Chanson P, Laws E, Schlechte J, Vance ML, Ho K, Giustina A \& Acromegaly Treatment Consensus Workshop Participants. Guidelines for acromegaly management: an update. Journal of Clinical Endocrinology and Metabolism 2009 94 1509-1517. (doi:10.1210/jc.2008-2421)

10 Biermasz NR, Pereira AM, Neelis KJ, Roelfsema F \& Romijn JA. Role of radiotherapy in the management of acromegaly. Expert Review of Endocrinology and Metabolism 20061 449-460. (doi:10.1586/ 17446651.1.3.449)

11 Swords FM, Monson JP, Besser GM, Chew SL, Drake WM, Grossman AB \& Plowman PN. Gamma knife radiosurgery: a safe and effective salvage treatment for pituitary tumours not controlled despite conventional radiotherapy. European Journal of Endocrinology 2009161 819-828. (doi:10.1530/EJE-09-0493)

12 Barrande G, Pittino-Lungo M, Coste J, Ponvert D, Bertagna X, Luton JP \& Bertherat J. Hormonal and metabolic effects of radiotherapy in acromegaly: long-term results in 128 patients followed in a single center. Journal of Clinical Endocrinology and Metabolism 2000 85 3779-3785. (doi:10.1210/jc.85.10.3779)

13 Epaminonda P, Porretti S, Cappiello V, Beck-Peccoz P, Faglia G \& Arosio M. Efficacy of radiotherapy in normalizing serum IGF-I, acid-labile subunit (ALS) and IGFBP-3 levels in acromegaly. Clinical Endocrinology 200155 183-189. (doi:10.1046/j.13652265.2001.01294.x)

14 Jenkins PJ, Bates P, Carson MN, Stewart PM \& Wass JA. Conventional pituitary irradiation is effective in lowering serum growth hormone and insulin-like growth factor-I in patients with acromegaly. Journal of Clinical Endocrinology and Metabolism 2006 91 1239-1245. (doi:10.1210/jc.2005-1616)

15 Attanasio R, Epaminonda P, Motti E, Giugni E, Ventrella L, Cozzi R, Farabola M, Loli P, Beck-Peccoz P \& Arosio M. Gamma-knife radiosurgery in acromegaly: a 4-year follow-up study. Journal of Clinical Endocrinology and Metabolism $2003 \mathbf{8 8} 3105-3112$. (doi:10.1210/jc.2002-021663)

16 Castinetti F, Taieb D, Kuhn JM, Chanson P, Tamura M, Jaquet P, Conte-Devolx B, Régis J, Dufour H \& Brue T. Outcome of gamma knife radiosurgery in 82 patients with acromegaly: correlation with initial hypersecretion. Journal of Clinical Endocrinology and Metabolism 2005 90 4483-4488. (doi:10.1210/jc.2005-0311)

17 Losa M, Gioia L, Picozzi P, Franzin A, Valle M, Giovanelli M \& Mortini P. The role of stereotactic radiotherapy in patients with growth hormone-secreting pituitary adenoma. Journal of Clinical Endocrinology and Metabolism 200893 2546-2552. (doi:10. 1210/jc.2008-0135)

18 Ronchi CL, Attanasio R, Verrua E, Cozzi R, Ferrante E, Loli P, Montefusco L, Motti E, Ferrari DI, Giugni E, Beck-Peccoz P \& Arosio M. Efficacy and tolerability of gamma knife radiosurgery in acromegaly: a 10-year follow-up study. Clinical Endocrinology 200971 846-852. (doi:10.1111/j.1365-2265.2009.03589.x)

19 Swearingen B, Barker FG II, Katznelson L, Biller BM, Grinspoon S, Klibanski A, Moayeri N, Black PM \& Zervas NT. Long-term mortality after transsphenoidal surgery and adjunctive therapy for acromegaly. Journal of Clinical Endocrinology and Metabolism 1998 83 3419-3426. (doi:10.1210/jc.83.10.3419)

20 Jaffrain-Rea ML, Minniti G, Moroni C, Esposito V, Ferretti E, Santoro A, Infusino T, Tamburrano G, Cantore G \& Cassone R. Impact of successful transsphenoidal surgery on cardiovascular risk factors in acromegaly. European Journal of Endocrinology 2003 148 193-201. (doi:10.1530/eje.0.1480193)

21 Ronchi CL, Varca V, Beck-Peccoz P, Orsi E, Donadio F, Baccarelli A, Giavoli C, Ferrante E, Lania A, Spada A \& Arosio M. Comparison between six-year therapy with long-acting somatostatin analogs and successful surgery in acromegaly: effects on cardiovascular risk factors. Journal of Clinical Endocrinology and Metabolism 2006 91 121-128. (doi:10.1210/jc.2005-1704)

22 Colao A, Auriemma RS, Galdiero M, Lombardi G \& Pivonello R. Effects of initial therapy for five years with somatostatin analogs for acromegaly on growth hormone and insulin-like growth factor-I levels, tumor shrinkage, and cardiovascular disease: a prospective study. Journal of Clinical Endocrinology and Metabolism 200994 3746-3756. (doi:10.1210/jc.2009-0941)

23 Mazziotti G, Floriani I, Bonadonna S, Torri V, Chanson P \& Giustina A. Effects of somatostatin analogs on glucose homeostasis: a metaanalysis of acromegaly studies. Journal of Clinical Endocrinology and Metabolism 200994 1500-1508. (doi:10. 1210/jc.2008-2332)

24 Brada M, Ashley S, Ford D, Traish D, Burchell L \& Rajan B. Cerebrovascular mortality in patients with pituitary adenoma. Clinical Endocrinology $2002 \mathbf{5 7} 713-717$. (doi:10.1046/j.13652265.2002.01570.x)

25 Sherlock M, Reulen RC, Alonso AA, Ayuk J, Clayton RN, Sheppard MC, Hawkins MM, Bates AS \& Stewart PM. ACTH deficiency, higher doses of hydrocortisone replacement, and radiotherapy are independent predictors of mortality in patients with acromegaly. Journal of Clinical Endocrinology and Metabolism 200994 4216-4223. (doi:10.1210/jc.2009-1097)

26 Rosén T \& Bengtsson BA. Premature mortality due to cardiovascular disease in hypopituitarism. Lancet 1990336 285-288. (doi:10.1016/0140-6736(90)91812-O)

27 Tomlinson JW, Holden N, Hills RK, Wheatley K, Clayton RN, Bates AS, Sheppard MC \& Stewart PM. Association between premature mortality and hypopituitarism. West Midlands Prospective Hypopituitary Study Group. Lancet 2001357 425-431. (doi:10.1016/S0140-6736(00)04006-X)

28 Ben-Shlomo A. Pituitary gland: predictors of acromegaly-associated mortality. Nature Reviews. Endocrinology 20106 67-69. (doi:10. 1038/nrendo.2009.267) 
29 Giustina A, Barkan A, Casanueva FF, Cavagnini F, Frohman L, Ho K, Veldhuis J, Wass J, Von Werder K \& Melmed S. Criteria for cure of acromegaly: a consensus statement. Journal of Clinical Endocrinology and Metabolism 200085 526-529. (doi:10.1210/ jc. 85.2.526)

30 Ronchi CL, Varca V, Giavoli C, Epaminonda P, Beck-Peccoz P, Spada A \& Arosio M. Long-term evaluation of postoperative acromegalic patients in remission with previous and newly proposed criteria. Journal of Clinical Endocrinology and Metabolism 200590 1377-1382. (doi:10.1210/jc.2004-1974)

31 Ronchi CL, Ferrante E, Rizzo E, Giavoli C, Verrua E, Bergamaschi S, Lania AG, Beck-Peccoz P \& Spada A. Long-term basal and dynamic evaluation of hypothalamic-pituitary-adrenal (HPA) axis in acromegalic patients. Clinical Endocrinology 200869 608-612. (doi:10.1111/j.1365-2265.2008.03270.x)

32 Ronchi CL, Giavoli C, Ferrante E, Verrua E, Bergamaschi S, Ferrari DI, Corbetta S, Montefusco L, Arosio M, Ambrosi B, Spada A \& Beck-Peccoz P. Prevalence of GH deficiency in cured acromegalic patients: impact of different previous treatments. European Journal of Endocrinology 2009161 37-42. (doi:10.1530/ EJE-09-0222)

33 Matthews DR, Hosker JP, Rudenski AS, Naylor BA, Treacher DF \& Turner RC. Homeostasis model assessment: insulin resistance and beta-cell function from fasting plasma glucose and insulin concentrations in man. Diabetologia 198528 412-419. (doi:10. 1007/BF00280883)

34 Expert Panel on Detection, Evaluation, and Treatment of high blood cholesterol in Adults. Executive summary of the third report of the national cholesterol education program (NCEP) expert panel on detection, evaluation, and treatment of high blood cholesterol in adults (ATPIII). Journal of the American Medical Association 2001 287 356-359.

35 Jermendy G, Hidvegi T, Hetyesi K \& Biro L. Is it time to use the new lower limit of impaired fasting glucose (IFG) among the ATP III criteria for diagnosis of the metabolic syndrome? Prevalence rate of the metabolic syndrome using old and new lower limits of IFG in obese and/or hypertensive subjects Diabetes, Nutrition and Metabolism 200417 169-170.

36 Giustina A, Chanson P, Bronstein MD, Klibanski A, Lamberts S, Casanueva FF, Trainer P, Ghigo E, Ho K \& Melmed S. Consensus statement: a consensus on criteria for cure of acromegaly. Journal of Clinical Endocrinology and Metabolism 201095 3141-3148. (doi:10.1210/jc.2009-2670)

37 Peacey SR, Toogood AA, Veldhuis JD, Thorner MO \& Shalet SM. The relationship between 24-hour growth hormone secretion and insulin-like growth factor I in patients with successfully treated acromegaly: impact of surgery or radiotherapy. Journal of Clinical Endocrinology and Metabolism 200186 259-266. (doi:10.1210/ jc.86.1.259)

38 Filipsson H, Monson JP, Koltowska-Häggström M, Mattsson A \& Johannsson G. The impact of glucocorticoid replacement regimens on metabolic outcome and comorbidity in hypopituitary patients. Journal of Clinical Endocrinology and Metabolism 200691 3954-3961. (doi:10.1210/jc.2006-0524)

39 Shih HA \& Loeffler JS. Radiation therapy in acromegaly. Reviews in Endocrine and Metabolic Disorders 20089 59-65. (doi:10.1007/ s11154-007-9065-x)

40 Castinetti F, Régis J, Dufour H \& Brue T. Role of stereotactic radiosurgery in the management of pituitary adenomas. Nature Reviews. Endocrinology 20106 214-223. (doi:10.1038/nrendo.2010.4)

41 Melmed S. Acromegaly pathogenesis and treatment. Journal of Clinical Investigation 2009119 3189-3202. (doi:10.1172/ JCI39375)

42 Katznelson L. Approach to the patient with persistent acromegaly after pituitary surgery. Journal of Clinical Endocrinology and Metabolism 201095 4114-4123. (doi:10.1210/jc.2010-0670)

Received 19 January 2011

Accepted 7 February 2011 